\title{
Utilizando o Aplicativo Criptomática para Ensinar Conteúdos Matemáticos do Ensino Médio com Uso da Criptografia
}

Michel Ferreira Batista, FACAPE, Brasil, michelfbatista@ hotmail.com Ricardo Argenton Ramos, UNIVASF, Brasil, ricargentonramos@gmail.com Lucas Florêncio de Brito, UNIVASF, Brasil, lucasdibex@gmail.com

Resumo: Muitos alunos na educação básica possuem dificuldade no aprendizado de matemática, o uso de tecnologias computacionais pode ser uma alternativa para ajudar a contornar essa situação. Este artigo objetiva apresentar os resultados do desenvolvimento e utilização do aplicativo "Criptomática", concebido para possibilitar aos alunos aprenderem e praticarem os conteúdos dessa disciplina no ensino médio relacionados com a temática da criptografia de Hill. A validação foi feita com o foco em obter o ponto de vista de utilizadores e especialistas da área de exatas. Os resultados mostram que o aplicativo atendeu significativamente a proposta estabelecida de apoiar o ensino e aprendizagem de alguns conteúdos matemáticos. Possibilitando a participação ativa da formulação das respostas com uso dessa ferramenta.

Palavras-chave: Matemática, Ensino e aprendizagem. Aplicativo, Criptografia.

\section{Using The Cryptomatic Application To Teach High School Math Contents Using Cryptography}

Abstract: A several numbers of students from basic education have difficulty learning math, thus, the use of computer technology may be an alternative to help this situation. This article aims to present the results of the development and use of the "Cryptomatic" mobile application, designed to assist students to learn and practice contents of the high school mathematics discipline related to Hill's cryptography. This mobile Application was applied in a validation to obtain the view point of users and specialists of the math area. The results show that the application has significantly met the proposal goal to support the teaching and learning of some mathematical content. It enables the active participation of the formulation of the responses using this tool.

Keywords: Mathematics, Teaching and learning, Application, Encryption.

\section{Introdução}

O ensino e aprendizagem de matemática são de extrema importância, tanto quanto a alfabetização, para o desenvolvimento social e a formação profissional de uma pessoa, uma vez que a capacidade de estabelecer o raciocínio lógico matemático nas atividades cotidianas, como: passar um troco, realizar uma compra com desconto, efetuar medições; são triviais para que um indivíduo conquiste sua independência (LESEUX; NETO; DARSIE, 2017). A matemática também pode ser encontrada em várias outras áreas do conhecimento humano, desempenhando aplicações práticas capazes de desenvolver habilidades e competências para a formação cognitiva dos alunos (SANTOS et al., 2017).

Atualmente, muitos alunos da educação básica, incluindo o ensino médio, possuem dificuldade de aprendizagem desta disciplina. Segundo os dados disponibilizados da última pesquisa (2017) do Sistema de Avaliação da Educação Básica (SAEB), cerca de 71,67 \% dos alunos têm nível insuficiente de aprendizado, 23,81\% apresentam nível básico e apenas 4,52\% mostraram estar no nível adequado de 
conhecimento (BRASIL, 2017). Esses dados mostram a deficiência do ensino da matemática no cenário brasileiro.

Algumas das formas de contornar o cenário negativo no aprendizado matemático são através do uso de tecnologias computacionais, em que estão inclusos os softwares e aplicativos, possibilitando a integração da matemática com outras disciplinas. Assim, será possível de mudar a forma de realização dos exercícios de matemática e despertar a curiosidade de outras áreas, evidenciam Masola e Allevato (2016). Komar et al. (2017) corroboram no sentido que a matemática deve ser trabalhada em conjunto com outras áreas, como Psicologia, Sociologia e Filosofia

Uma área que pode ser explorada para o ensino da matemática é a da criptografia, um dos campos de estudo da segurança da informação e com o crescimento do uso da Internet está cada vez mais presente no dia-dia das pessoas, tornando-se assim, indispensável para garantir a confiabilidade de processos cotidianos. A cifra de Hill é um método matemático riquíssimo utilizado para codificação de mensagens. Groenwald e Olgin (2011) destacam que utilizar esse método nas aulas de matemática possibilita exercitar diversos conteúdos matemáticos simultaneamente, como números primos, álgebra modular, congruência, matrizes; presentes no $2^{\circ}$ ano do ensino médio ( $11^{\circ}$ ano), mais precisamente no eixo de (Álgebra e funções), presentes no currículo de matemática do ensino médio. Outros autores também destacam o uso da cifra de Hill como ferramenta eficiente para o ensino de matemática (BARBOSA; CORNELISSEN, 2017).

Diante desse exposto, este trabalho pretende responder algumas perguntas:

Como possibilitar aos alunos, do ensino médio, meios acessíveis para a prática e interação da matemática aprendida em sala de aula, envolvendo uma temática que pode ser aplicável no seu dia-dia e que traga significância para os conteúdos aprendidos?

O quanto o aplicativo Criptomática pode auxiliar os alunos em relação a motivação, experiência do usuário de jogos e no seu aprendizado em matemática?

Portanto, o objetivo do artigo é descrever a experiência de desenvolver e aplicar uma ferramenta de apoio ao ensino e aprendizagem de matemática, para a prática e exercício de conteúdos matemáticos específicos na temática da criptografia de Hill. Especificadamente, buscou-se abordar neste trabalho o diferencial do aplicativo desenvolvido para fins didáticos; modelo utilizado para avaliação de aplicativos educacionais e a validação do aplicativo por meio de questionário aplicado.

Este artigo está organizado como se segue: após esta introdução são apresentados os materiais e métodos usados na pesquisa. Na sequência, são apresentados os resultados e discussões, concluindo com as considerações finais e referências utilizadas.

\section{Materiais e Métodos}

Para alcançar os objetivos deste artigo foram utilizadas metodologias em duas partes distintas, a primeira foi referente a elaboração do aplicativo seguindo boas práticas da engenharia de software e atendendo as funcionalidades de cunho educacional. Já a segunda parte, usou um método para avaliar o aplicativo proposto com o foco na opinião de especialistas.

Fase 1: A criação do aplicativo móvel (nomeado Criptomática) seguiu o método de desenvolvimento proposto por Vaupel et al. (2018) e teve início através da análise de requisitos de aplicativos similares disponíveis em lojas de aplicativos. Além das propostas trazidas por esses aplicativos, buscou-se adicionar funcionalidades que acrescentam o viés de reforço do aprendizado, apontado como o principal problema no levantamento do SAEB (BRASIL, 2017).

Através da técnica de análise de competidores, foram encontrados 3 aplicativos similares (utilizam criptografia de Hill): Cryptography, Caesar e Decipher, e as suas 
funcionalidades foram comparadas, conforme Tabela 1. Alguns dos requisitos considerados foram: a utilização do Idioma Português Brasileiro, a possibilidade de o usuário praticar os cálculos matemáticos no próprio aplicativo e a apresentação de informações de como realizar esses cálculos através de dicas, fortalecendo assim o aprendizado do aluno.

Tabela 1 - Comparativo entre aplicativos similares na temática Criptografia de Hill.

\begin{tabular}{|l|c|c|c|c|}
\hline & Cryptography & Caesar & Decipher & Criptomática \\
\hline Idioma Nativo (Português Brasileiro) & NÃO & NÃO & NÃO & SIM \\
\hline Utiliza a cifra de Hill & SIM & SIM & SIM & SIM \\
\hline Usuário participa dos cálculos & NÃO & NÃO & NÃO & SIM \\
\hline Traz dicas para realizar cada etapa & NÃO & NÃO & NÃO & SIM \\
\hline $\begin{array}{l}\text { Expõe conteúdo Matemático para } \\
\text { consulta }\end{array}$ & NÃO & SIM & NÃO & SIM \\
\hline Interage com mensagens de aviso & SIM & NIM \\
\hline Salva a criptografia em arquivo & SIM & SIM & SIM \\
\hline $\begin{array}{l}\text { Exporta a mensagem criptografada para } \\
\text { rede social, SMS ou e-mail; }\end{array}$ & SIM \\
\hline
\end{tabular}

Fonte: Os autores (2018)

Os requisitos funcionais foram levantados observando as etapas envolvidas no processo de cifrar e decifrar mensagens listados nos trabalhos de Pereira (2015), Brandão (2017), Costa e Caetano (2017). Também foi considerada à proposta de trazer para o usuário uma bagagem teórica matemática. Os Requisitos não-funcionais foram levantados baseados nos conceitos de interação, segurança e satisfação do usuário como: a validação dos campos de entrada, campos nulos, campos com restrições de tipos de entrada, filtros de valores permitidos, mensagens de alerta informando condições não permitidas e mensagens de alerta que aguardam confirmação do usuário (auxilio ao erro).

As ferramentas utilizadas nesta etapa foram as disponíveis pelo framework Ionic3 (https://ionicframework.com). Este se destina a criação de aplicações híbridas para dispositivos móveis, permitindo desenvolver uma única vez para múltiplas plataformas. A vantagem considerada para o uso deste framework neste trabalho foram os recursos do CSS, HTML e JSON que facilita o desenvolvimento de aplicativos.

Fase 2: $\mathrm{O}$ experimento teve a população escolhida com a intenção de se obter a perspectiva de usuários especialistas, como proposto no trabalho de Dias et al. (2013). Assim, foram selecionados 06 usuários que trabalham ou estudam na área de exatas. A quantidade dos participantes se baseia em selecionar participantes perto do ideal apontado no trabalho de Nielsen (2000) que afirma que os melhores resultados de avaliação de usabilidade são obtidos através de testes com cinco usuários.

A operacionalização do experimento foi feita com os seguintes passos:

1 - Preenchimento dos questionários. Todos os participantes foram convidados a responderem os questionários para fim de verificação do nível de conhecimento anterior ao desenvolvimento da oficina do aplicativo. Os questionários foram feitos com base no trabalho de Savi et al. (2011), detalhado logo mais nesta seção.

2 - Participação em aula de equalização de conhecimento dos participantes. Foi dada uma aula para apresentar os objetivos do projeto, como seria realizada a avaliação, sobre o tema criptografia de Hill e as operações matemáticas utilizadas para o processo de cifrar e decifrar mensagens. Essa aula teve a duração de 30 minutos.

3 - Instalação e utilização do aplicativo. O aplicativo em questão foi disponibilizado para os participantes no intuito de que eles realizem os devidos testes. Nada foi explicado aos participantes sobre o funcionamento do aplicativo, somente foi dado o passo a passo para a instalação do aplicativo no celular de cada participante. $\mathrm{O}$ tempo de utilização foi de 60 minutos. 
4 - Preenchimento dos questionários. Após o fim da etapa anterior, os participantes foram solicitados a responderem novamente os questionários para fim de coletar informações sobre o conhecimento adquirido.

5 - Avaliação dos resultados. Com a posse dos questionários preenchidos foram utilizados os métodos estatísticos (detalhados no decorrer desta seção) e chegou-se as conclusões apresentadas na seção a seguir.

O método de avaliação de jogos educacionais utilizado neste trabalhado foi adaptado do elaborado por Savi et al. (2011), buscando medir o grau de atendimento do aplicativo ao objetivo proposto pelo projeto: ensinar o aluno as operações matemáticas que o capacitam para realizar a temática da criptografia de Hill.

Por meio de questionário previamente elaborado, os participantes validaram temas sobre o aplicativo. Além dessas questões, duas questões foram colocadas no final do questionário para medir qualitativamente o aplicativo. Nela, os participantes poderiam apontar 3 pontos fortes do aplicativo e sugestões de melhoria. Essas questões representam as revisões dos usuários do aplicativo. Esse mecanismo de feedback será comum quando o aplicativo estiver disponível em um repositório online.

Para validar o aplicativo foi empregado o questionário de jogos educacionais elaborado por Savi et al. (2011), composto pelos modelos de treinamento de Kirkpatrick, modelo ARCS de avaliação motivacional, a avaliação de experiência do usuário (User eXperience - UX) e taxonomia BLOOM voltada para os objetivos de aprendizagem, conforme ilustra a Figura 1.

A avaliação referente as subescalas de motivação, experiência do usuário e aprendizagem foi utilizada a escala de Likert, com numeração que varia de 1 a 5 . Para cada afirmação proposta o usuário escolhe um valor que melhor representasse sua opinião quanto a sua observação sobre o aplicativo. A numeração atribuída indica que para valores próximos de 1 o usuário "discorda fortemente" e 5 "concorda fortemente". Na avaliação dos objetivos de aprendizagem referentes a taxonomia BLOOM (lembrar, compreender e aplicar), utilizou-se o teste de hipótese $t$ Student bilateral para amostras pareadas a fim de observar se houve diferença média na percepção do nível de conhecimento entre o antes e o depois da realização do jogo.

Figura 1: Estrutura do modelo de avaliação de jogos educacionais

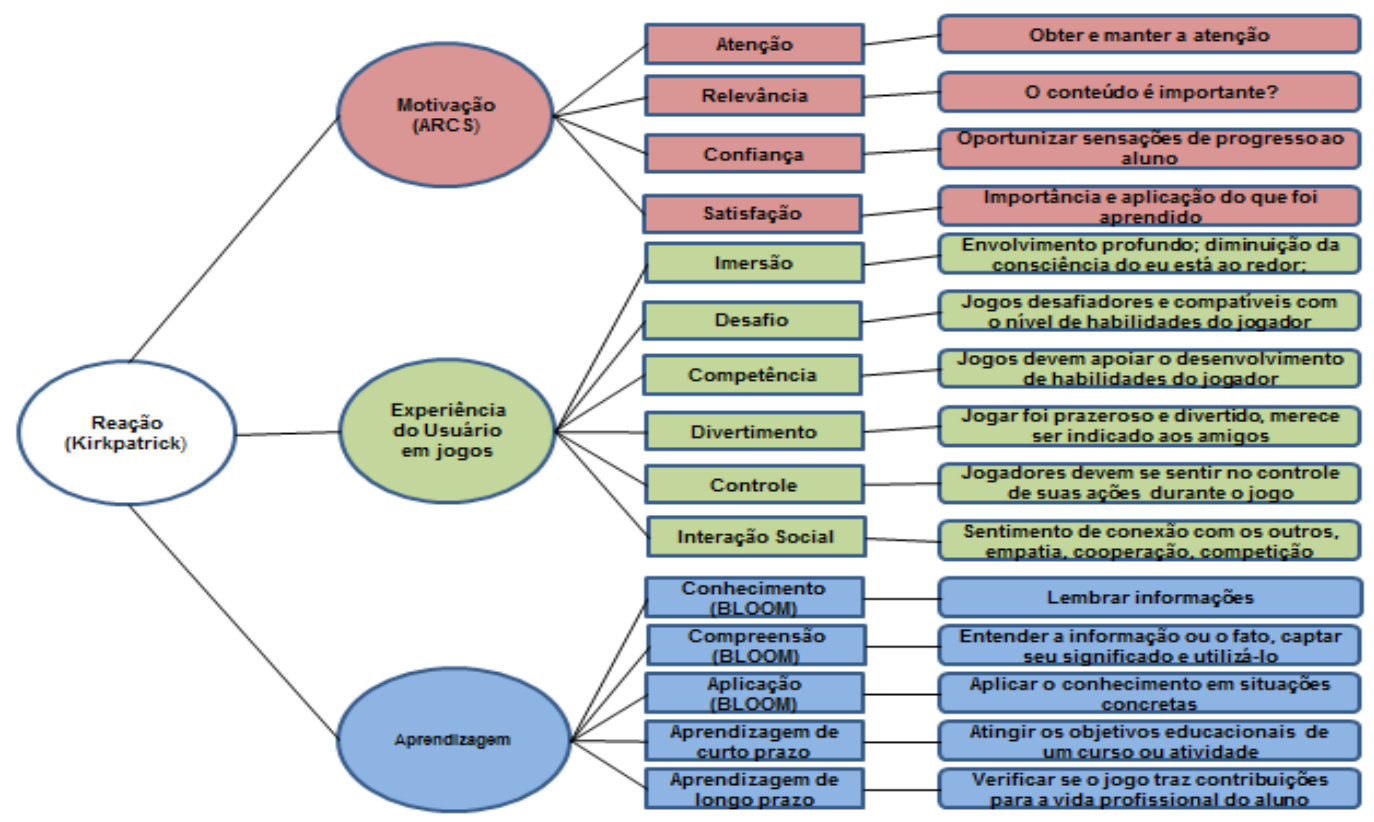

Fonte: Savi et al. (2011) 


\section{Resultados e discussões}

Algumas das telas do aplicativo Criptomática podem ser vistas na Figura 1. A primeira tela (a esquerda) apresenta a tela inicial do aplicativo onde o usuário poderá escolher entre praticar a criptografia ou então conhecer alguns conteúdos matemáticos referentes ao tema. Na tela do meio é apresentado um menu e na tela a direita da Figura 2 é apresentada a primeira etapa de como o usuário fará a criptografia de uma mensagem.

Figura 2 - Telas do aplicativo Criptomática
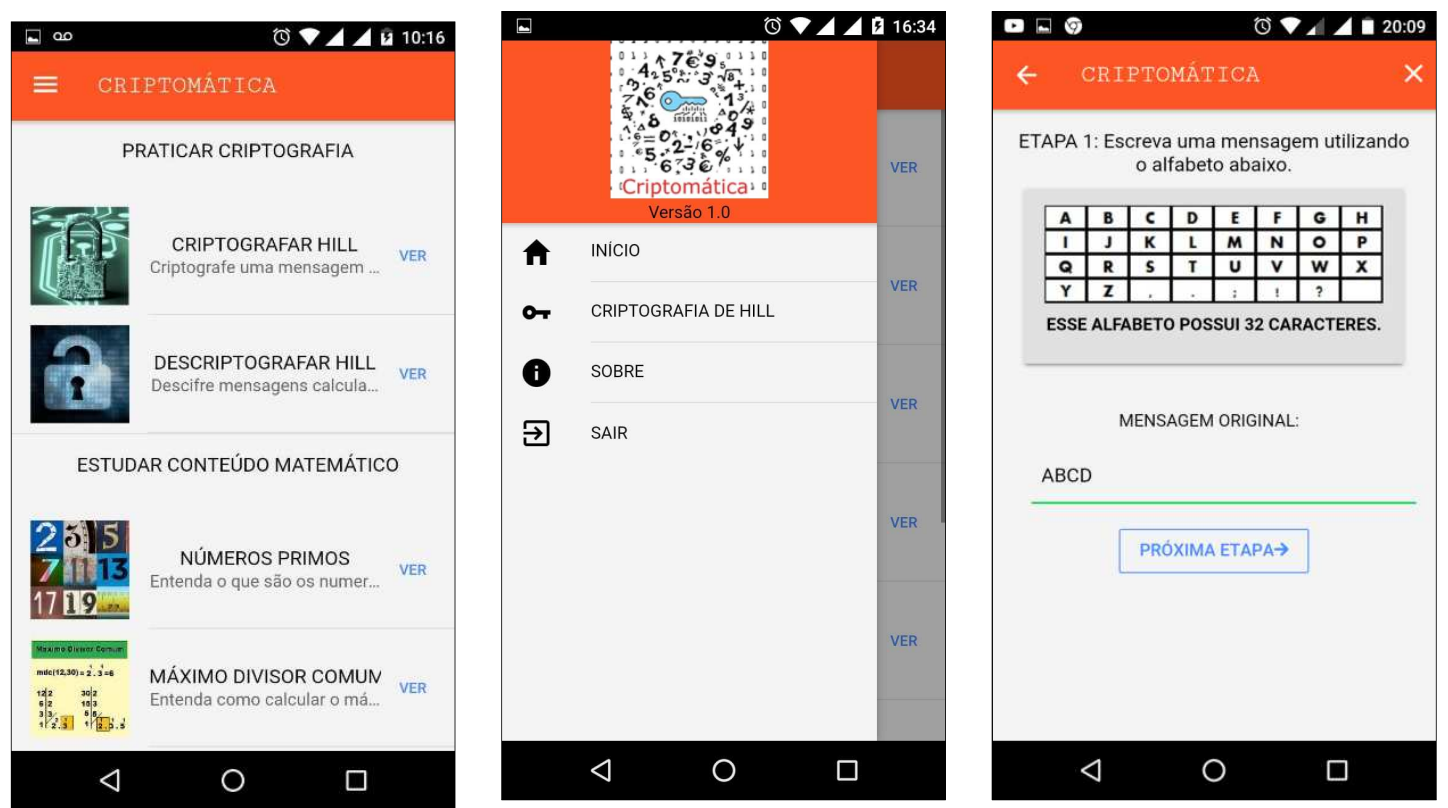

Fonte: Os autores (2018)

A seguir, serão apresentados os resultados alcançados após a avaliação feita pelos participantes da oficina, referente a cada subescala do modelo de avaliação adaptado de Savi et al. (2011).

Subescala Motivação: De modo geral o item motivação não apresentou nenhuma nota 1, o que caracterizaria discordar plenamente de alguma afirmação. Trouxe resultados expressivos quanto a satisfação do usuário e a relevância do conteúdo trabalhado no aplicativo conforme mostra o Gráfico 1.

Dimensão atenção: Se destacou na captura da atenção dos participantes no início da aplicação com $83 \%$ de aceitação, mostrando que a tela inicial pode ter contribuído. $O$ design do aplicativo foi bem avaliado com $67 \%$ de aprovação e $17 \%$ sugeriram melhorias, já a variação de conteúdos teve $66 \%$ que consideraram ter ajudado a manter a atenção no aplicativo.

Dimensão Relevância: $84 \%$ dos usuários consideraram que o conteúdo do aplicativo possuía conexão com seus conhecimentos prévios e $83 \%$ que os conteúdos abordados eram relevantes para o interesse deles. 66\% concordaram que conseguem aprender com a forma de abordagem dos conteúdos pelo aplicativo.

Dimensão Confiança: Mostrou que 100\% dos usuários sentiram estar aprendendo ao desenvolver as etapas propostas pelo aplicativo. E 50\% acharam fácil entender o aplicativo. Destes 33\% sentiram alguma dificuldade em interagir, o que de acordo com as sugestões observadas remete a perceber que o conteúdo matemático trabalhado teve que ser pesquisado com maior frequência na guia de "dicas" e "consulta ao conteúdo", para então poder cumprir as etapas. Houve sugestões para utilizar vídeo aulas com os conteúdos e até diminuir o número de etapas. 
Dimensão Satisfação: Em destaque, 100\% dos pesquisados afirmaram que se sentiram satisfeitos por saber que poderão aplicar na prática o que aprenderam no aplicativo. $66 \%$ reconhecem que por esforço próprio conseguem concluir as etapas propostas pelo aplicativo, entretanto $17 \%$ não acharam que foi pelo seu esforço próprio.

Gráfico 1: Resultado subescala Motivação

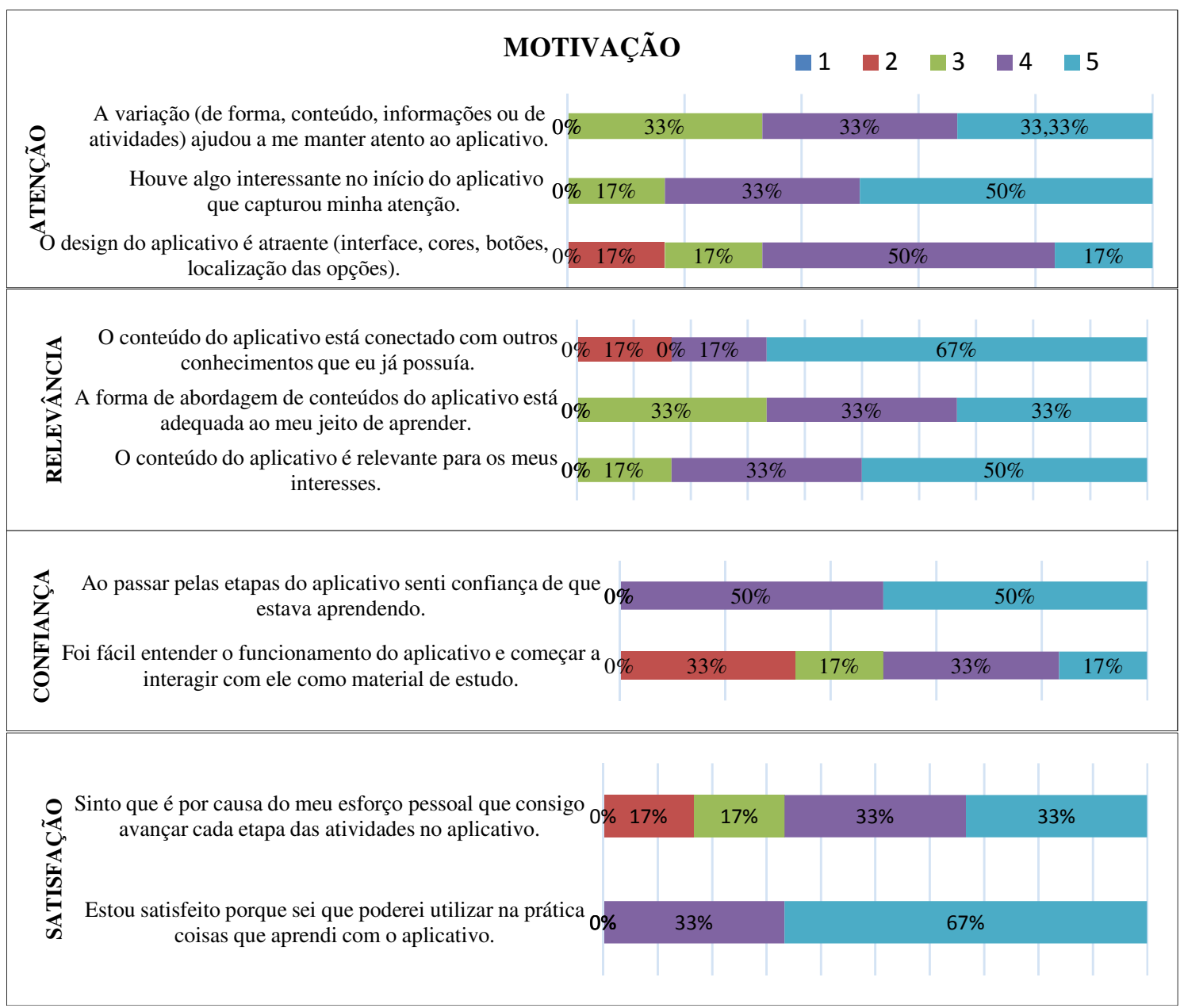

Fonte: Os autores (2018)

Subescala Experiência do Usuário: Quanto a experiência do usuário, as únicas discordâncias apresentadas foram no quesito "imersão", onde $17 \%$ dos usuários disseram que não se sentiram imergidos durante as atividades e $33 \%$ que não se concentraram no aplicativo. Nos demais quesitos houve avaliações positivas com $100 \%$ de aprovação quanto ao divertimento, desafios propostos pelo aplicativo e a auto avaliação de competência para alcançar os objetivos propostos pelo aplicativo, conforme Gráfico 2.

Dimensão Imersão: Neste quesito as opiniões ficaram divididas sobre a capacidade de concentração no aplicativo, 33\% concordam e 33\% não concordam, os outro $33 \%$ concordam parcialmente. $50 \%$ não perceberam o tempo passar durante a realização das atividades, enquanto $17 \%$ não demostraram esta concentração. $34 \%$ imergiram por completo no ambiente do aplicativo, contra $33 \%$ que sentiram mais dispersos e externos ao mundo real.

Dimensão Interação social: A maioria considera com 66\% que o aplicativo promove momentos de cooperação e competição entre os colegas que utilizam e que sentem vontade de compartilhar as tarefas que desempenham no aplicativo (criptografar e decifrar). $33 \%$ consideram interagir com outros colegas afim de solucionar etapas do aplicativo, os outros $50 \%$ concordaram parcialmente nisso. 
Dimensão Desafio: Aqui 100\% dos usuários julgam terem se divertido com o aplicativo e que o nível de desafio proposto pelo aplicativo é adequadamente desafiador para eles. Enquanto $83 \%$ sentiram vontade de tentar novas possibilidades com o aplicativo.

Dimensão Divertimento: $100 \%$ dos pesquisados gostariam de utilizar o aplicativo novamente, sendo $83 \%$ o recomendaria para algum colega, $17 \%$ concordam parcialmente.

Dimensão Competência: $100 \%$ se consideraram competentes por sentirem eficiência ao avançarem nas etapas propostas do aplicativo. Já $66 \%$ consideram terem atingido os objetivos do aplicativo por mérito próprio, $33 \%$ concordam parcialmente.

\section{Gráfico 2: Resultado subescala Experiência do Usuário}

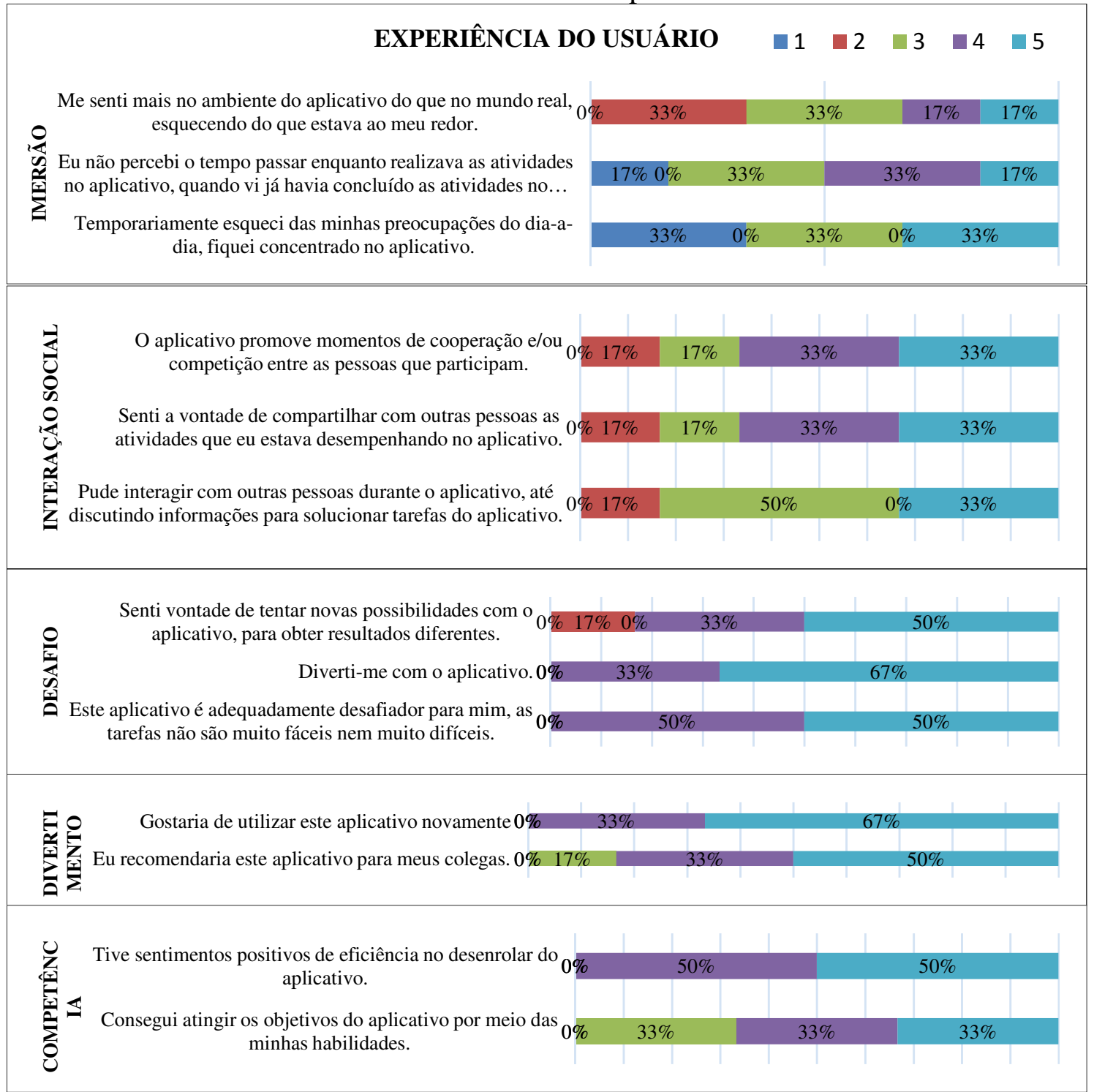

Fonte: Os autores (2018)

Subescala Aprendizagem: Essa subescala não apresentou nenhuma discordância quanto ao que foi perguntado e mostrou mais de $2 / 3$ dos participantes concordando com a eficiência do aplicativo em termos de aprendizagem e de sua contribuição para um bom desempenho futuro profissional, conforme Gráfico 3.

Dimensão aprendizagem em curto prazo: $84 \%$ dos pesquisados concordam que o aplicativo demonstrou eficiência no aprendizado dos conteúdos apresentados, $17 \%$ 
concordam em parte. $67 \%$ são a favor de que o aplicativo contribuiu para aprendizagem dos conteúdos matemáticos.

Dimensão aprendizagem em longo prazo: $66 \%$ tem o sentimento que o aplicativo veio somar em termos de contribuição para o desempenho na vida profissional, enquanto $33 \%$ concordam parcialmente com isso.

Gráfico 3: Resultado subescala Aprendizagem

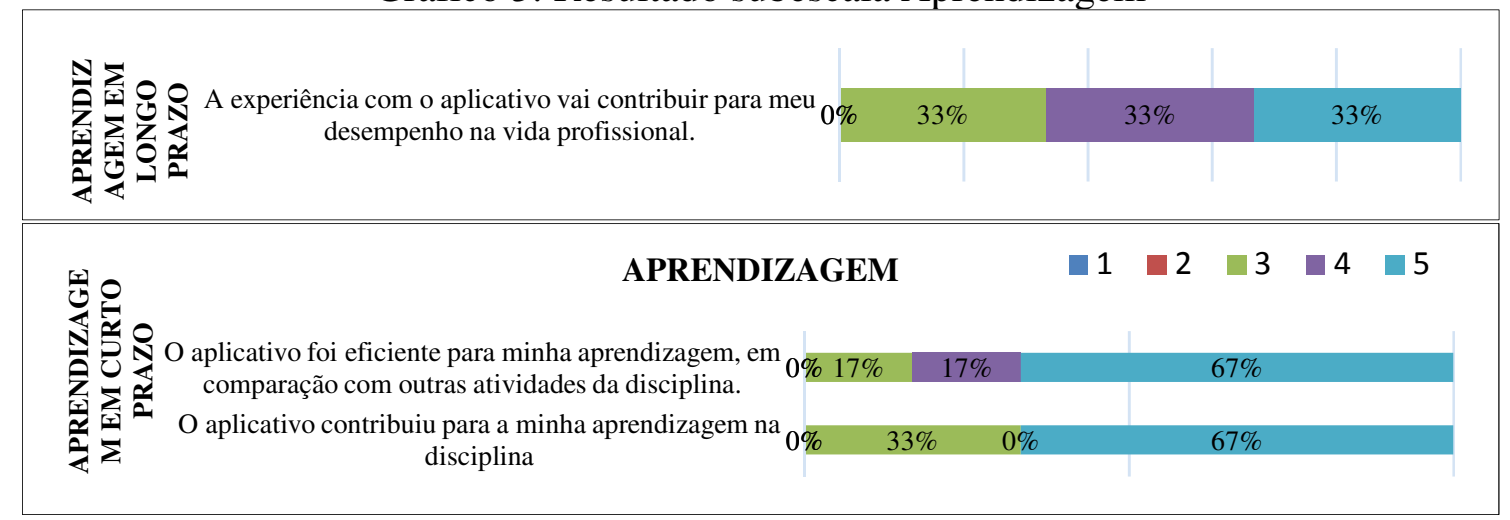

Fonte: Os autores (2018)

Avaliação dos objetivos de aprendizagem: $\mathrm{O}$ Gráfico 4 apesenta o resultado das médias obtidas pela avaliação dos participantes em relação à aprendizagem sobre os temas e conteúdos matemáticos abordados antes e depois da aplicação da oficina do aplicativo. De modo geral, observa-se que houve significativo ganho de conhecimento em praticamente todos conteúdos matemáticos abordados após o uso do aplicativo. A partir desses dados foi calculado o teste $t$ Student para obter um resultado mais aproximado de averiguar o nível de eficiência do aplicativo na aprendizagem desses conteúdos.

Gráfico 4: Média aprendizagem dos conteúdos antes e depois do aplicativo.

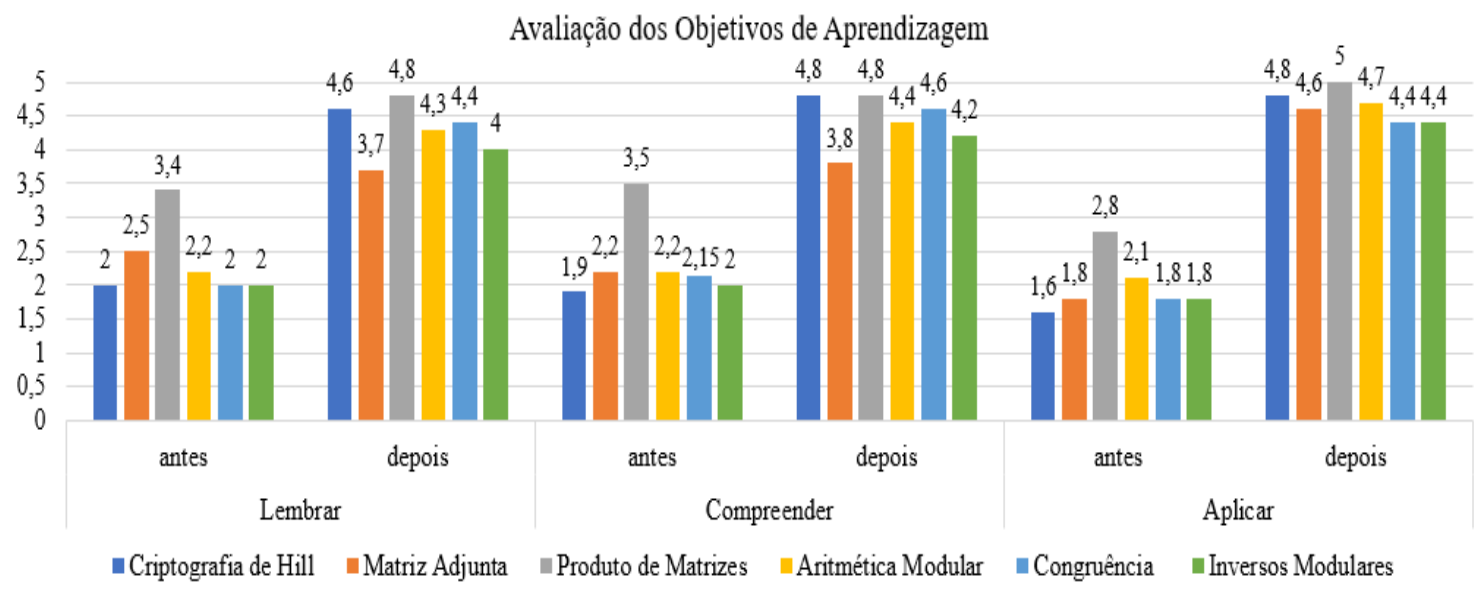

Fonte: Os autores (2018)

$\mathrm{O}$ valor calculado de $t$ e o valor-p referente aos conteúdos matemáticos analisados antes e depois do uso do aplicativo para cada objetivo de aprendizagem (BLOOM) foram calculados e apresentados na Tabela 2.

Houve aumento significativo para o conteúdo de Criptografia de Hill, Aritmética modular, congruência e inversos modulares tanto para lembrar, compreender e aplicar. Em relação a matriz adjunta só houve aumento significativo na percepção da aprendizagem em relação a aplicar e para produto de matrizes apenas quanto a compreender. Isso se justifica porque a diferença entre as notas antes e depois desses 
conteúdos eram pequenas, ou seja, esses participantes (por serem especialistas na área) já compreendiam bem esse conceito, logo não havia muito o que acrescentar de conhecimento pelo aplicativo.

Tabela 2: Resultado teste t Student

\begin{tabular}{|c|c|c|c|c|c|c|}
\hline & \multicolumn{2}{|c|}{ Lembrar } & \multicolumn{2}{c|}{ Compreender } & \multicolumn{2}{c|}{ Aplicar } \\
\hline & $\mathrm{T}$ & valor-p & $\mathrm{T}$ & valor-p & $\mathrm{T}$ & $\mathrm{p}$-valor \\
\hline Criptografia de Hill & $-3,833$ & $0,018^{*}$ & $-3,713$ & $0,02^{*}$ & $-5,488$ & $0,005^{*}$ \\
\hline Matriz adjunta & $-2,449$ & 0,07 & $-2,666$ & 0,056 & $-4,802$ & $0,008^{*}$ \\
\hline $\begin{array}{c}\text { Produto de } \\
\text { Matrizes }\end{array}$ & $-2,333$ & 0,079 & $-2,081$ & $0,01^{*}$ & $-2,75$ & 0,051 \\
\hline $\begin{array}{c}\text { Aritmética } \\
\text { Modular }\end{array}$ & $-2,806$ & $0,048^{*}$ & $-2,993$ & $0,04^{*}$ & $-3,833$ & $0,018^{*}$ \\
\hline Congruência & $-3,538$ & $0,024^{*}$ & $-3,296$ & $0,03^{*}$ & $-3,474$ & $0,025^{*}$ \\
\hline $\begin{array}{c}\text { Inversos } \\
\text { Modulares }\end{array}$ & $-2,828$ & $0,047^{*}$ & $-29,938$ & $0,04^{*}$ & $-3,474$ & $0,025^{*}$ \\
\hline
\end{tabular}

*Estatisticamente significativo

Fonte: Os autores (2018)

\section{Considerações finais}

Com base no levantamento bibliográfico realizado no presente trabalho, observouse que a educação matemática necessita cada vez mais de iniciativas na produção de ferramentas de apoio ao ensino e aprendizagem. As dificuldades na disciplina são reais e estão presentes no cenário educacional com maior abrangência no ensino médio, conforme mostraram os dados do SAEB (2017). O uso de aplicativos é uma ótima alternativa para a aprendizagem, pois ensina ao mesmo tempo que atrai os alunos para atividades lúdicas. A temática da criptografia de Hill, justifica para o aluno onde e como a matemática é empregada no campo profissional estando presente em nosso cotidiano. Mudar a forma de realizar atividades de matemática, desperta a curiosidade e interesse dos alunos, uma vez que rompe com a forma engessada e tradicional de resolver problemas matemáticos.

Conforme objetivos levantados no início do trabalho, podemos inferir que o aplicativo Criptomática atendeu com sucesso às condições propostas, sendo bem avaliado pelos participantes nas subescalas de motivação, experiência do usuário e aprendizagem. Os resultados obtidos mostraram que o aplicativo desenvolvido difere dos seus concorrentes por possuir funcionalidades que apoiam o usuário durante $\mathrm{o}$ desenvolvimento das atividades de criptografia e permitem ao mesmo participar da formulação das respostas, além de estar no idioma Português brasileiro. O modelo utilizado para avaliação de aplicativo educacional destacou o nível de satisfação e o aprendizado dos avaliados. A partir dos dados do teste t Student pode-se concluir que o aplicativo foi eficiente para o ensino de Criptografia de Hill, aritmética modular, congruência e inversos modulares.

\section{REFERÊNCIAS}

BARBOSA, Lucas; CORNELISSEN, Mariana Garabini. Cifra de Hill: Uma Aplicação ao Estudo de Matrizes. RECEN-Revista Ciências Exatas e Naturais, v. 19, n. 2, p. 152167, 2017 
BRANDÃO, Mariana Martins Durães. Uma adaptação da cifra de Hill para estudo de matrizes. Dissertação (Mestrado) - Universidade Federal de Ouro Preto. - 2017. 91f. Ouro Preto-MG.

BRASIL. MEC/INEP. Relatório do Sistema Nacional de Avaliação da Educação Básica (SAEB) - ciclo 1990. Brasília, 2017.

COSTA, Edson Marques; CAETANO, Natalia Gonçalves. Criptografia com utilização de cifra de Hill e cifra afim. Revista eletrônica matemática e estatística em foco. Vol5.N1. julho-2017. Disponível em: <http://www.seer.ufu.br/index.php/ matematicaeestatisticaemfoco/article/view/33777/20752>

DIAS, J. M. et al. Avaliação de jogos educacionais digitais baseada em Perspectivas. Uma experiência através do Jogo-simulador Kimera. Anais do Simpósio Brasileiro de Jogos e Entretenimento Digital, v. 1, p. 1, 2013.

GROENWALD, Claudia Lisete Oliveira; DE ASSIS OLGIN, Clarossa. Criptografia e o Currículo de matemática no Ensino Médio. Revista de Educação Matemática, v. 13, n. 15, p. 69-78, 2011.

KOMAR, Marcelo Fabricio Chociai et al. A modelagem matemática como metodologia para o ensino e a aprendizagem dos fractais. RENOTE - Revista Novas Tecnologias na Educação . v. 15, n. 2 (2017). ISSN 1679-1916. Disponível em: <https://seer.ufrgs.br/renote/article/view/79262>

LESEUX, Sandro Luiz; NETO, Orestes Zivieri; DARSIE, Marta Maria Pontin. Aprendizagem matemática nos seis primeiros anos da educação básica no município de Comodoro-MT. EDUCA - Revista Multidisciplinar em Educação, Porto Velho, v.4, n.7, p. 2-23, jan/abr, 2017. Disponível em: < http://www.periodicos.unir.br/ index.php/EDUCA/index>. ISSN: 2359-2087.

MASOLA, Wilson de Jesus; ALlEVATO, Norma Suely Gomes. Dificuldades de aprendizagem matemática de alunos ingressantes na educação superior. REBES Rev. Brasileira de Ensino Superior, 2(1): 64-74, jan.-mar. 2016. ISSN 24473944.NIELSEN J. Why You Only Need to Test with 5 Users, 2000. Disponivel em: <https://www.nngroup.com/articles/why-you-only-need-to-test-with-5-users/>.Acesso em 15 ago. 2018

PEREIRA, Nádia Marques Ikeda. Criptografia: uma nova proposta de ensino de matemática no ciclo básico. Dissertação (Mestrado) - Universidade Estadual Paulista "Júlio De Mesquita Filho". 2015.78f. Ilha Solteira - SP.

SANTOS, Elaine Estaneck Rangel dos et al.. A linguagem matemática e seus reflexos na aprendizagem de alunos: uma experiência nas turmas de ensino médio da nova educação de jovens e adultos (neja).InterdisciplinaryScientificJournal v.4, n.3, p. 226245, Jul-Set, 2017. ISSN: 2358-8411.

SAVI, Rafael; WANGENHEIM, C.; BORGATTO, A. Um Modelo de Avaliação de Jogos Educacionais na Engenharia de Software. Anais do XXV Simpósio Brasileiro de Engenharia de Software (SBES 2011), São Paulo, 2011.

VAUPEL, Steffen et al. Model-driven development of mobile applications for Android and iOS supporting role-based app variability. Software \& Systems Modeling, v. 17, n. 1, p. 35-63, 2018. 